\title{
Encuesta sobre el manejo de injertos del ligamento cruzado anterior contaminados accidentalmente
}

\section{Survey on management of accidentally contaminated anterior cruciate ligament grafts}

\author{
Francisco Figueroa David Figueroa Rafael Calvo Alejandro Vaisman ${ }^{\circledR}$ Felipe Pino ${ }^{(1)}$ \\ Rafael Calvo Mena Luis A. O’Connell ${ }^{\odot}$ \\ ${ }^{1}$ Departamento de Cirugía Ortopédica, Clínica Alemana de Santiago- \\ Universidad del Desarrollo Medical School, Santiago, Chile \\ Address for correspondence Luis A. O'Connell, MD, Servicio de \\ Traumatología y Ortopedia de Adultos, Clínica Alemana de Santiago- \\ Rev Chil Ortop Traumatol 2019;60:86-90. \\ Universidad del Desarrollo, Av Manquhue Norte 1410, Vitacura, \\ Santiago, Chile (e-mail: luis.oconnell@gmail.com).
}

\section{Resumen \\ Palabras clave \\ - ligamento cruzado anterior \\ - reconstrucción \\ - injerto \\ - contaminación \\ - manejo \\ - clorhexidina}

Abstract
Objetivo Encuestar a cirujanos de rodilla en Chile, sobre su conducta en contaminaciones accidentales del injerto, en reconstrucciones de ligamento cruzado anterior (LCA).

Métodos Se realizó una encuesta anónima en relación a la incidencia, tratamiento y resultados clínicos de los injertos de LCA contaminados, a todos los médicos pertenecientes al área de traumatología y ortopedia asistentes al congreso Ateneo de rodilla 2015.

Resultados Se encuestaron 54 médicos, 33 (61\%) especialistas en cirugía de rodilla y 21 (39\%) médicos en formación. De los cirujanos de rodilla, 15 (45\%) reportaron al menos un caso de contaminación, de los cuales 12 (80\%) informaron un único evento, 3 (20\%) indicaron la eventualidad en 2, 3 y 5 ocasiones respectivamente, para un total de 22 injertos contaminados. De los cirujanos que reportaron injertos contaminados, 11 (73\%) realizan 50 o más reconstrucciones al año. La decisión tomada en los casos de contaminación fue en 13 oportunidades (59\%), lavar el injerto y utilizarlo de acuerdo al plan inicial; en 6 casos (27\%), utilizar aloinjerto; en 2 ocasiones (9\%), utilizar autoinjerto alternativo; y en 1 oportunidad (5\%); triplicar el semitendinoso. Ninguno de los casos de contaminación reportó infecciones.

Conclusiones En casos de una contaminación accidental, la mayoría de los cirujanos encuestados prefieren optar por la descontaminación del injerto y su utilización en la reconstrucción de acuerdo al plan inicial; para lo cual la clorhexidina y antibióticos sería la solución a usar de preferencia.

Nivel de Evidencia Nivel V, opinión de experto.

Objective To survey knee surgeons who perform anterior cruciate ligament (ACL) reconstruction in Chile, about the management when $\mathrm{ACL}$ graft contamination occurs. Methods An anonymous survey was conducted to all physicians belonging to the area of traumatology and orthopedics who attended the 2015 annual Chilean Knee Society received

January 15, 2019 accepted September 10, 2019
DOI https://doi.org/

$10.1055 / \mathrm{s}-0039-1698794$ ISSN $0716-4548$.
Copyright $\odot 2019$ by Thieme Revinter

Publicações Ltda, Rio de Janeiro, Brazil
License terms

()(1) $\Theta \circledast$ 


\author{
Keywords \\ - anterior cruciate \\ ligament \\ - reconstruction \\ - graft \\ - contamination \\ - management \\ - chlorhexidine
}

meeting. The survey questioned the incidence, treatment, and outcomes of ACL graft contaminations.

Results Fifty-four physicians were surveyed, of which 33 (61\%) where knee surgeons and 21 (39\%) physicians in training. Of the 33 knee surgeons, 15 (45\%) reported at least one contamination during their career. Of those $15,12(80 \%)$ had one event, and three surgeons (20\%) informed having 2, 3, and 5 episodes respectively, for a total of 22 reported contaminated grafts. Of the surgeons who reported a contaminated graft, 11 (73\%) performed 50 or more $\mathrm{ACL}$ reconstructions annually. In 13 opportunities (59\%) the management for a contaminated graft was cleansing the graft and proceeding as planned. In 6 cases (27\%) an allograft was used, in 2 occasions (9\%) a different graft was harvested, and in 1 opportunity (5\%) a semitendinosus graft triplication was performed. No infections in any of the contaminated grafts were reported.

Conclusions In case of accidental graft contamination during an $\mathrm{ACL}$ reconstruction, knee surgeons most often preferred disinfecting the graft and using it as the initial plan.

Level of Evidence Level V, expert opinion.

\section{Introducción}

La reconstrucción del ligamento cruzado anterior (LCA) es un procedimiento común, con tasas reportadas de 32 a 45 por 100,000 habitantes. Las infecciones desarrolladas posterior a una cirugía del LCA son una complicación rara, con incidencia menor al $2 \%,{ }^{1-4}$ que acarrea posibles consecuencias catastróficas para el procedimiento, siendo la contaminación accidental del injerto el posible evento desencadenador. Si bien no existen datos sobre la incidencia de estos eventos, Izquierdo y col., ${ }^{5}$ reportó que el $25 \%$ de los cirujanos especialistas en medicina del deporte, tuvo al menos un incidente de contaminación accidental del injerto.

Posiblemente por ser la contaminación accidental del injerto un evento poco frecuente, no ha sido posible la creación y publicación de guías clínicas. Según nuestro conocimiento, a la fecha no se han reportado protocolos, resultados o experiencia clínica local sobre el manejo de injertos contaminados accidentalmente en cirugías de LCA. Este estudio es una encuesta sobre los eventos y actitudes tomadas por especialistas en cirugía de rodilla con práctica clínica en Chile.

\section{Métodos}

Se empleó una modificación de la encuesta utilizada por Izquierdo y col., ${ }^{5}$ para realizar un cuestionario anónimo a todos los médicos pertenecientes al área de traumatología y ortopedia asistentes al congreso Ateneo de rodilla 2015 (-Figura 1). El objetivo de la encuesta, es obtener datos sobre la incidencia de injertos contaminados accidentalmente durante cirugías de LCA, la conducta tomada y los resultados clínicos de esos eventos durante la práctica clínica de los especialistas en cirugía de rodilla. También se analizaron las decisiones predilectas de médicos especialistas, sobre casos hipotéticos de contaminación accidental del injerto en una cirugía de LCA.

\section{Métodos estadísticos}

Los datos fueron estudiados por medio de análisis estadísticos descriptivos.

\section{Resultados}

En total fueron encuestados 54 médicos, de los cuales 33 (61\%) eran especialistas en cirugía de rodilla, 21 (39\%) traumatólogos que a la fecha realizaban su entrenamiento en la especialidad de cirugía de rodilla o en traumatología y ortopedia.

El promedio de años de experiencia entre los especialistas fue de 16 años (rango 2-30 años), con una media de 44 (rango 10-100) reconstrucciones de LCA realizadas anualmente.

De los 33 cirujanos de rodilla encuestados, 15 (45\%) reportaron al menos un caso de contaminación accidental durante una cirugía de reconstrucción del LCA. El 73\% (11 cirujanos) de los especialistas que reportaron haber tenido un evento de contaminación accidental, indicaron realizar 50 o más cirugías de LCA al año. De los eventos reportados, el $80 \%$ ( 12 cirujanos) reportaron un evento único, mientras que 3 cirujanos (20\%) indicaron la eventualidad en 2, 3 y 5 ocasiones respectivamente.

Un total de 22 injertos contaminados fueron reportados por 15 cirujanos; de los cuales 13 (59\%) injertos fueron lavados y utilizados en la reconstrucción de acuerdo al plan inicial. En 6 casos (27\%), se optó por la utilización de un aloinjerto, en tanto que en 2 ocasiones (9\%) se utilizó un autoinjerto alternativo como remplazo del injerto contaminado, y en 1 oportunidad (5\%) se decidió triplicar el injerto semitendinoso (-Figura 2).

En los casos donde hubo contaminación accidental del injerto y se eligió lavar y usar el injerto de acuerdo al plan inicial, no se reportaron incidentes de infección postoperatoria.

En caso de lavar el injerto, de los 33 cirujanos especialistas en cirugía de rodilla 18 (55\%) refirieron el uso de clorhexidina 


\section{Categoría \\ a) Staff \\ b) Fellow \\ c) Becado (Residente) \\ d) Médico general}

\section{Preguntas para Staff}

1. Años de experiencia

\section{Reconstrucciones anuales de LCA}

3. ¿Alguna vez se a caído o contaminado el injerto ?

(De ser afirmativa su respuesta anotar el número de veces)

a. Sí

b. No

4. ¿ Qué hizo ? (Responder únicamente si la respuesta a la pregunta 3 fue afirmativa)

a. Lo limpió y lo usó

b. Tomó otro autoinjerto

c. Usó aloinjerto

d. Otro (Por favor detallar)

5. ¿ Desarrolló infección ? (Responder únicamente si respondió que limpió y uso el injerto)

a. $\mathrm{Si}$

b. No

\section{Preguntas para todos}

7. En caso de que se cayera o contaminara el injerto, ¿Qué haría?

a. Limpiarlo y usarlo

b. Tomar otro autoinjerto

c. Usar aloinjerto

d. Otro (Por favor detallar)

8. Si decidiera lavarıo, ¿Qué opción escogería?

a. Solución con clorhexidina

b. Solución con antibiótico

c. Solución fisiológica

d. Solución con povidona

e. Combinación de clornexidina y antibiótico

\section{Abreviaciones: LCA Ligamento Cruzado Anterior.}

Fig. 1 Encuesta sobre el manejo de injertos contaminados accidentalmente en reconstrucciones de Ligamento Cruzado Anterior en Chile.

$\mathrm{y}$ antibióticos, 5 (15\%) prefirieron utilizar únicamente clorhexidina, 5 (15\%) una solución con antibióticos, 4 (12\%) lavar con solución fisiológica, y 1 (3\%) cirujano escogió emplear una solución con povidona (- Figura 3).

\section{Discusión}

La contaminación accidental del injerto durante una cirugía de reconstrucción del LCA, plantea un problema sobre qué hacer en esa situación. Debido a que es un evento poco frecuente, existe muy poca información en la literatura para poder recomendar el protocolo de manejo más adecuado.

En base a nuestros datos, 15 de 33 (45\%) cirujanos especialistas en cirugía de rodilla, reportaron un total de 22 injertos contaminados de forma accidental, de los cuales 13 (59\%) procedieron a lavarlos y continuar con la cirugía como planeada de forma inicial.

Nuestros datos contrastan de manera importante con los reportados por Izquierdo y col., quien en una encuesta realizada a especialistas en medicina del deporte informó 


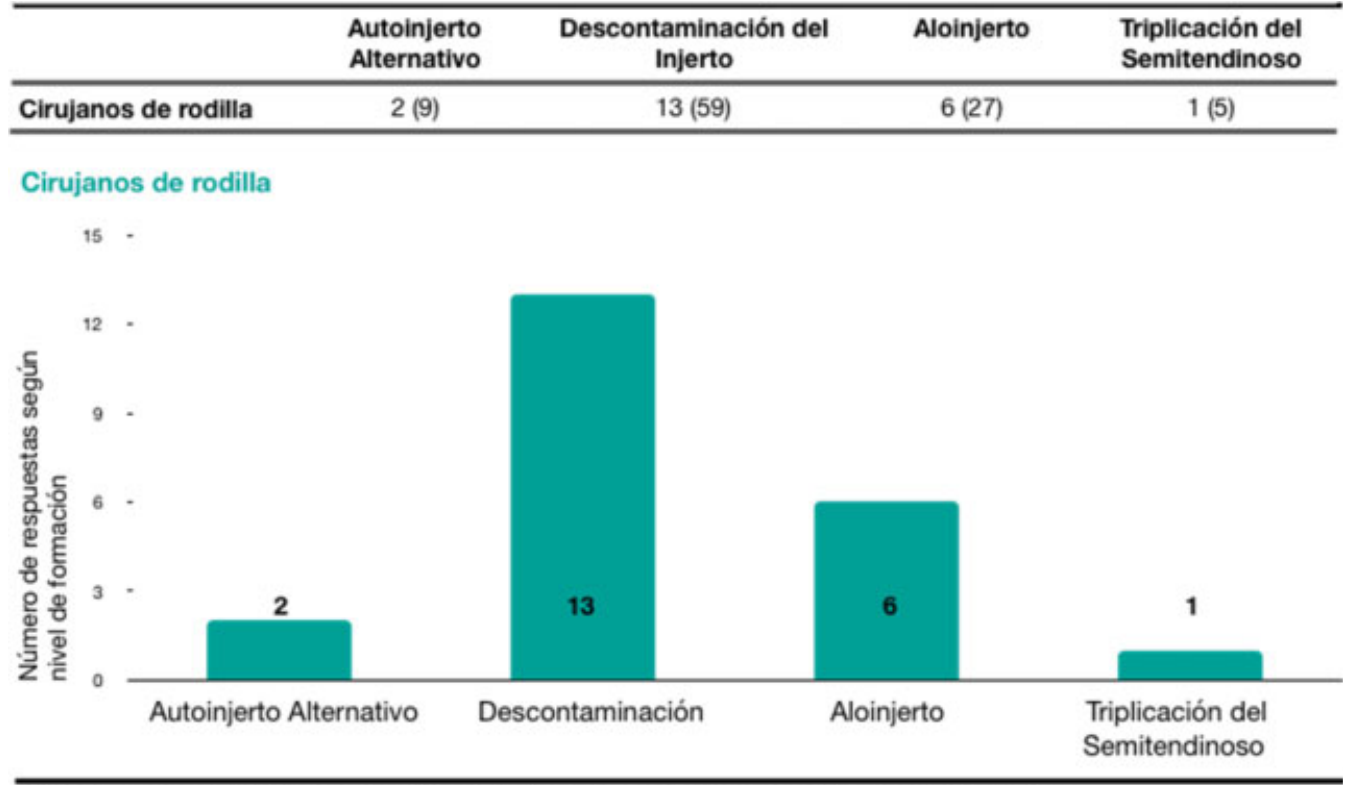

Abreviaciones: LCA Ligamento Cruzado Anterior. Los datos se muestran cómo n (\%).

Fig. 2 Respuesta acerca del manejo de injertos de LCA contaminados.

\begin{tabular}{lccccc}
\hline & $\begin{array}{c}\text { Clorhexidina } \\
\mathbf{y} \\
\text { Antibióticos }\end{array}$ & Clorhexidina & Antibióticos & Fisiológica & Povidona \\
\hline Cirujanos de rodilla & $18(55)$ & $5(15)$ & $5(15)$ & $4(12)$ & $1(3)$ \\
\hline
\end{tabular}

Cirujanos de rodilla

20 .

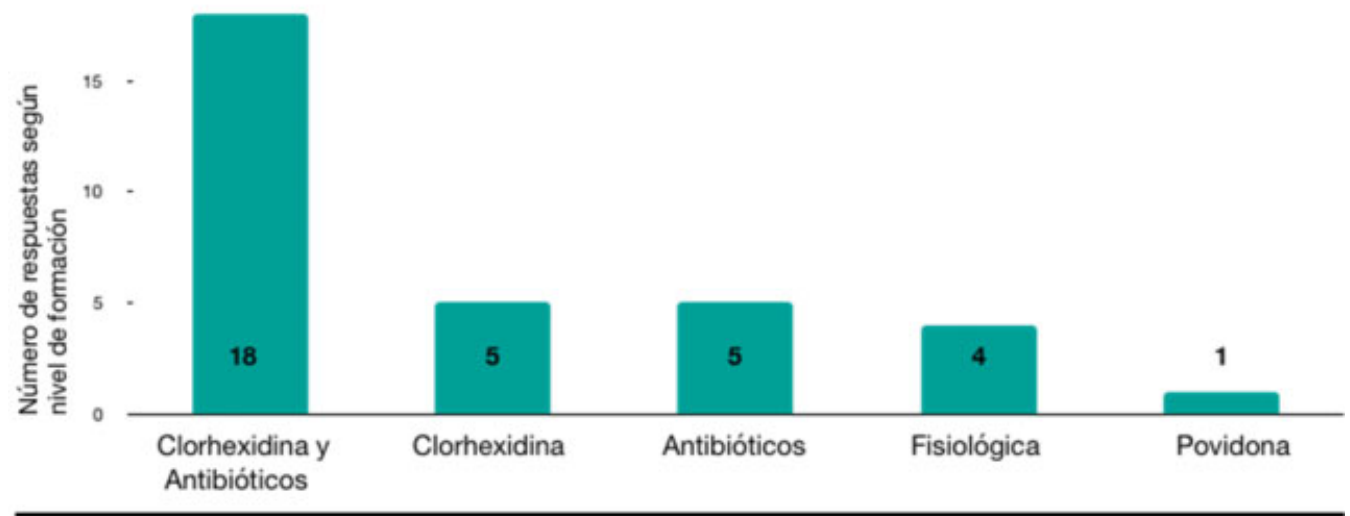

Los datos se muestran cómo n (\%).

Fig. 3 Solución de preferencia en caso de lavar el injerto.

que 49 de 196 (25\%) cirujanos refirieron 57 casos de contaminación, de los cuales 43 (75\%) tomaron la decisión de lavar el injerto y utilizarlo en la cirugía como planificado de forma inicial.

Usando los criterios descritos por Eddy, ${ }^{6}$ podemos asumir que entre los especialistas en cirugía de rodilla con práctica clínica en Chile existe poco acuerdo, menor al 60\%, sobre el manejo de injertos contaminados por accidente durante las cirugías de reconstrucción de LCA. Esta baja concordancia, únicamente permite plantear el lavado del injerto como una opción terapéutica. De forma contraria, la concordancia del 75\% alcanzada entre los especialistas de medicina del deporte estudiada por zquierdo y col. ${ }^{5}$ permite clasificar el lavado del injerto como una guía de práctica clínica.

Esta discrepancia en el manejo puede radicar en la escasa evidencia que existe al respecto de cómo desinfectar un injerto contaminado por accidente. Según nuestro conocimiento en la literatura, únicamente se han reportado 3 métodos que desinfectan el injerto en un $100 \%{ }^{7}$ (1) Sumergir el injerto por 30 minutos en una solución de clorhexidina al 4\%, y posteriormente sumergirlo en una solución de antibióticos (gentamicina, clindamicina y polimixina) por 30 minutos más, ${ }^{8}$ (2) Sumergir el injerto en una solución de $100 \mathrm{cc}$ a base de 2 antibióticos (bacitracina y polimixina) y agitarlo 
suavemente por 15 segundos, luego descartar la solución y repetir el proceso 10 veces, ${ }^{9}$ (3) Lavado a presión intermitente con $3 \mathrm{~L}$ de clorhexidina al $4 \%$ ó $2 \%{ }^{10}$

La gran desventaja del primer protocolo, es que se trata de un proceso largo que demora 1 hora en realizarse. El método de agitación mecánica únicamente incluyó organismos cultivados en una sola sala quirúrgica, lo que permite sospechar que no sea una flora universalmente representativa, siendo esa posiblemente la causa del reporte de resultados diversos con el uso de esa técnica. ${ }^{8,11}$ En consecuencia, es posible asumir que el método de lavado a presión intermitente, es el manejo con mayor evidencia consistente de desinfección y el más práctico de implementar.

Al analizar la evidencia sobre la desinfección con clorhexidina, es importante resaltar que el efecto bacteriostático y bactericida es dependiente de la concentración a la que se use. ${ }^{12}$ Eso hace indispensable saber si a diferentes concentraciones de clorhexidina, existen repercusiones sobre las propiedades biomecánicas y bioquímicas de los injertos, las cuales pudiesen provocar complicaciones post operatorias diferentes al desarrollo de una infección.

Sobel y col., ${ }^{13}$ reportó que no encontró diferencias en las propiedades biomecánicas de aloinjertos patelares, posterior a un lavado de 30 minutos con clorhexidina al $4 \%$, al compararlas con un grupo control que se mantuvo húmedo con gasas mojadas en solución salina fisiológica. Resultados que concuerdan con lo reportado por Han y col., ${ }^{7}$ quien informó que el lavado a presión pulsátil de tendones de origen bovino con 3 litros de clorhexidina al 2\%, no debilita sus propiedades mecánicas de tracción.

Cabe destacar que ambos estudios evaluaron las propiedades biomecánicas inmediatamente posterior a terminar el protocolo de desinfección.

Con respecto al efecto que la clorhexidina ejerce sobre las propiedades bioquímicas del colágeno y fibroblastos, Alomar y col., ${ }^{14}$ reportó que el lavado a presión pulsátil con clorhexidina al $4 \%$, disocia el colágeno recién formado, mientras que el uso de clorhexidina al $2 \%$, no afecta su integridad. Con respecto a la viabilidad celular, sus resultados resaltan que el lavado pulsátil a concentraciones tan bajas como el $2 \%$, tiene efectos citotóxicos en los fibroblastos, lo cual provocaría que el injerto se comportase como un aloinjerto, necesitando más tiempo para su intengración y confiréndole un mayor riesgo de falla. ${ }^{15}$

\section{Conclusiones}

En caso de una contaminación accidental, la mayoría de los cirujanos encuestados, prefieren optar por la descontaminación del injerto y su utilización en la reconstrucción de acuerdo al plan inicial; para lo cual la clorhexidina y antibióticos serían la solución a usar de preferencia.
Conflicto de Intereses

Lo autor del trabajo declara no tener ningún conflicto de intereses.

\section{Bibliografía}

1 Frank M, Schmucker U, David S, Matthes G, Ekkernkamp A, Seifert J. Devastating femoral osteomyelitis after anterior cruciate ligament reconstruction. Knee Surg Sports TraumatolArthrosc 2008;16(01):71-74

2 Judd D, Bottoni C, Kim D, Burke M, Hooker S. Infections following arthroscopic anterior cruciate ligament reconstruction. Arthroscopy 2006;22(04):375-384

3 Mei-Dan O, Mann G, Steinbacher G, Ballester SJ, Cugat RB, Alvarez PD. Septic arthritis with Staphylococcus lugdunensis following arthroscopic ACL revision with BPTB allograft. Knee Surg Sports TraumatolArthrosc 2008;16(01):15-18

4 Schollin-Borg M, Michaëlsson K, Rahme H. Presentation, outcome, and cause of septic arthritis after anterior cruciate ligament reconstruction: a case control study. Arthroscopy 2003;19(09): 941-947

5 Izquierdo RJr, Cadet ER, Bauer R, Stanwood W, Levine WN, Ahmad CS. A survey of sports medicine specialists investigating the preferred management of contaminated anterior cruciate ligament grafts. Arthroscopy 2005;21(11):1348-1353

6 Eddy DM. Clinical decision making: from theory to practice. Designing a practice policy. Standards, guidelines, and options. JAMA 1990;263(22):3077-3084, 3081, 3084

7 Han Y, Giannitsios D, Duke K, Steffen T, Burman M. Biomechanical analysis of chlorhexidine power irrigation to disinfect contaminated anterior cruciate ligament grafts. Am J Sports Med 2011;39(07):1528-1533

8 Goebel ME, Drez D Jr, Heck SB, Stoma MK. Contaminated rabbit patellar tendon grafts. In vivo analysis of disinfecting methods. Am J Sports Med 1994;22(03):387-391

9 Parker RD, Maschke SD. Mechanical agitation and serial dilution: an option for anterior cruciate ligament graft sterilization. J Knee Surg 2008;21(03):186-191

10 Burd T, Conroy BP, Meyer SC, Allen WC. The effects of chlorhexidine irrigation solution on contaminated bone-tendon allografts. Am J Sports Med 2000;28(02):241-244http://www. ncbi.nlm.nih.gov/pubmed/10751002[Internet]

11 Molina ME, Nonweiller DE, Evans JA, Delee JC. Contaminated anterior cruciate ligament grafts: the efficacy of 3 sterilization agents. Arthroscopy 2000;16(04):373-378

12 Oosterwaal PJM, Mikx FHM, van den Brink ME, Renggli HH. Bactericidal concentrations of chlorhexidine-digluconate, amine fluoride gel and stannous fluoride gel for subgingival bacteria tested in serum at short contact times. J Periodontal Res 1989;24 (02):155-160

13 Sobel AD, Hohman D, Jones J, Bisson LJ. Chlorhexidine gluconate cleansing has no effect on the structural properties of human patellar tendon allografts. Arthroscopy 2012;28(12):1862-1866. Doi: 10.1016/j.arthro.2012.05.888

14 Alomar AZ, Gawri R, Roughley PJ, Haglund L, Burman M. The effects of chlorhexidine graft decontamination on tendon graft collagen and cell viability. Am J Sports Med 2012;40(07): 1646-1653

15 Jackson DW, Corsetti J, Simon TM. Biologic incorporation of allograft anterior cruciate ligament replacements. Clin OrthopRelat Res. United States; 1996:126-33 